\title{
Auditory Working Memory Load Impairs Visual Ventral Stream Processing: Toward a Unified Model of Attentional Load
}

\author{
Jane Klemen ${ }^{1,2}$, Christian Büchel ${ }^{1}$, Mira Bühler ${ }^{3}$, Mareike M. Menz ${ }^{4}$, \\ and Michael Rose ${ }^{1}$
}

\begin{abstract}
Attentional interference between tasks performed in parallel is known to have strong and often undesired effects. As yet, however, the mechanisms by which interference operates remain elusive. A better knowledge of these processes may facilitate our understanding of the effects of attention on human performance and the debilitating consequences that disruptions to attention can have. According to the load theory of cognitive control, processing of task-irrelevant stimuli is increased by attending in parallel to a relevant task with high cognitive demands. This is due to the relevant task engaging cognitive control resources that are, hence, unavailable to inhibit the processing of task-irrelevant stimuli. However, it has also been demonstrated that a variety of types of load (perceptual and emotional) can result in a reduction of the processing of task-
\end{abstract}

\section{INTRODUCTION}

At any one point in time, the human brain is so inundated by incoming sensory information that the need arises to select relevant from irrelevant information for further processing. A clear understanding of normal attentional processes will facilitate the interpretation of behavior on a wide variety of everyday attention-demanding tasks. We concern ourselves in this article with the interfering effects of task-relevant working memory (WM) load on the processing of task-irrelevant stimuli.

By proposing a hybrid model of attentional selection, Lavie, Hirst, de Fockert, and Viding (2004) and Lavie (1995) have attempted to integrate findings in support of early (see, e.g., Treisman \& Geffen, 1967; Broadbent, 1958) and late (see, e.g., Duncan, 1980; Norman, 1968; Deutsch \& Deutsch, 1963) attentional selection. Initially, load theory concerned perceptual load (Lavie, 1995; Lavie \& Tsal, 1994) and posited that, so long as sufficient resources are available, all perceptual information, be it

\footnotetext{
${ }^{1}$ University Medical Centre Hamburg Eppendorf, Hamburg, Germany, ${ }^{2}$ Cardiff University Brain Research Imaging Centre, Cardiff, UK, ${ }^{3}$ Central Institute of Mental Health, Mannheim, Germany, ${ }^{4}$ University of Luebeck, Luebeck, Germany
}

irrelevant stimuli, suggesting a uniform effect of increased load irrespective of the type of load. In the present study, we concurrently presented a relevant auditory matching task $[n$-back working memory (WM)] of low or high cognitive load (1-back or 2-back WM) and task-irrelevant images at one of three object visibility levels $(0 \%, 50 \%$, or $100 \%)$. fMRI activation during the processing of the task-irrelevant visual stimuli was measured in the lateral occipital cortex and found to be reduced under high, compared to low, WM load. In combination with previous findings, this result is suggestive of a more generalized load theory, whereby cognitive load, as well as other types of load (e.g., perceptual), can result in a reduction of the processing of taskirrelevant stimuli, in line with a uniform effect of increased load irrespective of the type of load.

relevant or irrelevant, is processed; increasing the perceptual load of a relevant task limits the resources available to, and thus the processing of, irrelevant information. Thus, it follows that under low perceptual load, the locus of selective attention is late, whereas under high perceptual load it is early. Importantly, the processing of taskrelevant as well as task-irrelevant information caused by late selection leads to interference between these disparate inputs, an effect that is diminished by early selection, as processing of irrelevant information is reduced.

Behavioral evidence in support of perceptual load theory is vast (for a review, see Lavie, 1995; Lavie \& Tsal, $1994)$ and this has more recently been added to by neuroimaging studies that address the actual processing mechanisms. Effects in accord with load theory have been shown as early as primary visual cortex and grow more pronounced in higher-order visual areas (e.g., Schwartz et al., 2005; Rees, Frith, \& Lavie, 1997).

In addition to perceptual load, the effects of cognitive load have been studied. de Fockert, Rees, Frith, and Lavie (2001) used a dual-task design in which participants held in WM a string of ordered or randomly mixed digits (low or high WM load, respectively), followed by the categorization of visually presented names such as those of politicians or pop stars in the presence of distractor faces, 
and with each trial ending with a memory probe for the digits. Using fMRI, higher activity was found in brain areas involved in face processing when a distractor face was presented under high compared to low WM load, prompting the interpretation that high WM load had impaired cognitive control processes that should have led to the rejection of the face stimuli for further processing.

Lavie et al. (2004) showed that selective attention in a flanker task is more prone to interference when concurrent WM load is high than when it is low. Using the same dual-task design, the authors also directly compared the effects of perceptual (small vs. large search set) and WM (small vs. large memory set) load. They found that flanker interference was increased by low perceptual load or high WM load. Last, the authors also assessed the impact of dual- versus single-task coordination because all their studies of cognitive load had used a dual-task paradigm. The authors found a clear increase in distractor interference under the dual- compared to the single-task condition and interpreted this as further evidence that distractor interference increases under high cognitive load.

Thus, load theory was extended to include cognitive as well as perceptual load influences (Lavie et al., 2004). According to this extension, the effect of cognitive load is diametrically opposed to that of perceptual load. When low demands are placed upon cognitive control processes, these control processes are capable of inhibiting the processing of task-irrelevant input. If, however, high demands are placed upon cognitive control processes, these control processes are exhausted by the high demands and are thus no longer available to inhibit the processing of task-irrelevant input. Thus, in summary, low perceptual and high cognitive load should result in strong interference of task-irrelevant stimuli with the processing of task-relevant ones, whereas high perceptual and low cognitive load should yield the converse effect. Note that the locus of selection in the case of cognitive load is always a late one. Irrelevant stimuli are perceived; the question is whether cognitive control processes are capable of suppressing their interference with the processing of relevant stimuli.

Others have also studied the influence of cognitive load with conflicting results. Rose, Schmid, Winzen, Sommer, and Buchel (2005) studied the influence of visual WM load on the processing of task-irrelevant visual stimuli without the intermediate demands of a further (dual-)task. In their experiment, task-irrelevant images were presented at one of five object visibility levels: $0 \%, 25 \%, 50 \%, 75 \%$, or $100 \%$. Participants were instructed to ignore these images and focus on the relevant visual WM task, 1-back or 2-back WM. The modulatory impact of the two WM conditions upon the processing of the task-irrelevant stimuli at the five object visibility levels was assessed through three measures: by a surprise recognition task, by the fMRI signal in the lateral occipital complex (LOC), an area involved in object processing (Malach et al., 1995), and by ERPs. Contrary to what load theory would suggest, the authors found that the increase in BOLD activation with object visibility in the LOC was smaller under high than under low WM load. Similarly, the recognition rates for the task-irrelevant stimuli were reduced under high compared to low WM load and so were the amplitudes of the N1 and P1 ERP components. The authors conclude that WM load has a similar effect on the processing of taskirrelevant visual stimuli as perceptual load does, thereby contesting load theory.

In a variation of Rose et al.'s (2005) task, Bingel, Rose, Glascher, and Buchel (2007) added the factor of concurrent pain to the WM task and task-irrelevant images. It was found that activation in the LOC was reduced not only under high, compared to low, WM load, but also when a painful stimulus was delivered compared to no pain. Furthermore, it has been found that the processing of the task-irrelevant stimuli can also be manipulated by the emotional valence of the task-irrelevant images. In their study, Glascher, Rose, and Buchel (2007) chose the task-irrelevant images so that some images were emotionally neutral, whereas others had a high negative valence, that is, they were more task-relevant. It was found that the task-irrelevant images were processed less if the images were neutral, that is, when they were less relevant.

The pattern emerging from this line of research is one where various factors have similar effects on the processing of task-irrelevant stimuli. One can consequently pose the question whether a common mechanism underlies the effects of these seemingly disparate factors (perceptual load, WM load, pain, and emotion). In this view of load, the general effect of increased load of a relevant task is a reduction of processing of task-irrelevant items.

The aim of this study was to investigate whether WM load of a relevant task interferes with the processing of task-irrelevant stimuli in a manner consistent with load theory or our own previous findings and a more generalized view of load (Bingel et al., 2007; Glascher et al., 2007; Rose et al., 2005). In these previous studies, the task-relevant and task-irrelevant stimuli were presented to the same modality, namely, vision. This mode of presentation may have caused the task-relevant and taskirrelevant inputs to interfere with each other's processing at a perceptual level, which may have precluded the true effect of WM load. To overcome this possible confound, we adapted a previous study (Rose et al., 2005) and presented the two tasks to different sensory modalities. We also believe that our task design yields clearer results than previous dual-task paradigms (e.g., de Fockert et al., 2001). In our paradigm, we measure the impact of WM load on the processing of task-irrelevant stimuli, whereas in dual-task paradigms, WM load impacts on attentional control, which in turn influences perceptual and postperceptual processes. Hence, the interpretation of the primary effect of WM load in dual-task paradigms is difficult, if not impossible, to sustain. Last, we attempted to keep processing mechanisms simple and avoid their interacting with one another by using task-irrelevant stimuli 
rather than distractors, which interfere with the processing of the task-relevant stimuli.

Activity in the LOC has been linked to the recognition of visually presented objects (Malach et al., 1995). It has been found that activation in this area reduces with increasing scrambling of image content (Grill-Spector et al., 1998). Various studies have verified that activation in the LOC is related to object recognition rather than related processes, such as verbalization (Reinholz \& Pollmann, 2005) or visual attention (Grill-Spector, Kushnir, Hendler, $\&$ Malach, 2000). In the current experiment, task-irrelevant images were presented at three different object visibility levels $(0 \%, 50 \%$, or $100 \%)$, thus functionally defining the LOC. We aimed to investigate the effects of a relevant cognitive load task on the processing of the task-irrelevant images in the LOC. Thus, concurrently with the irrelevant images, a relevant auditory matching task ( $n$-back WM) was performed at one of two cognitive load levels (1-back vs. 2-back WM). One group of participants underwent fMRI while performing the task. Another group acted as a behavioral control group, for which electrooculograms (EOGs) were recorded to show that modulation of LOC activity could not be accounted for by eye movements. Both groups of participants also performed a surprise recognition task of the task-irrelevant visual stimuli subsequent to the main experiment (Figure 1).

In this experiment, we investigated the interference of a task-relevant auditory WM task with the processing of task-irrelevant images in the LOC. Increasing object visibility was anticipated to result in increased processing in the LOC, as previously shown (Grill-Spector et al., 1998). The cross-sensory interference to be observed in the LOC was anticipated to reflect previous unisensory results for WM load induced interference (Rose et al., 2005) as well as modulations of this by pain (Bingel et al., 2007) and emotional valence (Glascher et al., 2007), that is, we expected to observe decreased processing of the task-irrelevant images in the LOC under high compared to low WM load. Such a result would conform to the perceptual, rather than the WM, load effect reported by Lavie et al. (2004) and Lavie (1995). Although not central to load theory, we additionally expected to find a phasic (Rees, Frackowiak, \& Frith, 1997) modulation of the object visibility-induced increase in activity in the LOC as observed in our previous studies (Bingel et al., 2007; Glascher et al., 2007; Rose et al., 2005). Thus, high load should not lead to a tonic (Rees, Frackowiak, et al., 1997) decrease in activation in the LOC, but should interact with object visibility so that the more recognizable an object is, the less it is processed.

\section{METHODS}

\section{Participants}

Twelve participants took part in the fMRI experiment (mean age $=26$ years, range $=23-29$ years, 8 women , a further 13 in the behavioral control experiment, including EOG recordings (mean age $=27$ years, range $=21$ 35 years, 11 women). All participants were right-handed and had normal or corrected-to-normal vision. None of the participants had any known hearing deficit. The study was approved by the local ethics committee, all procedures were in compliance with national legislation and the Code of Ethical Principles for Medical Research Involving Human Subjects of the World Medical Association (Declaration of Helsinki), and informed written consent was given by all participants prior to the experiment.

\section{Design and Procedure}

\section{fMRI and EOG}

Participants performed an auditory matching task whose WM load was manipulated (1-back or 2-back WM) while viewing task-irrelevant images that were presented at one of three object visibility levels ( $0 \%, 50 \%$, or $100 \%)$, thus yielding six experimental conditions (see Figure 1). One group of participants underwent fMRI and another acted as a behavioral control group for which EOGs were recorded. Both groups of participants also performed a surprise recognition task of the task-irrelevant visual stimuli subsequent to the main experiment.

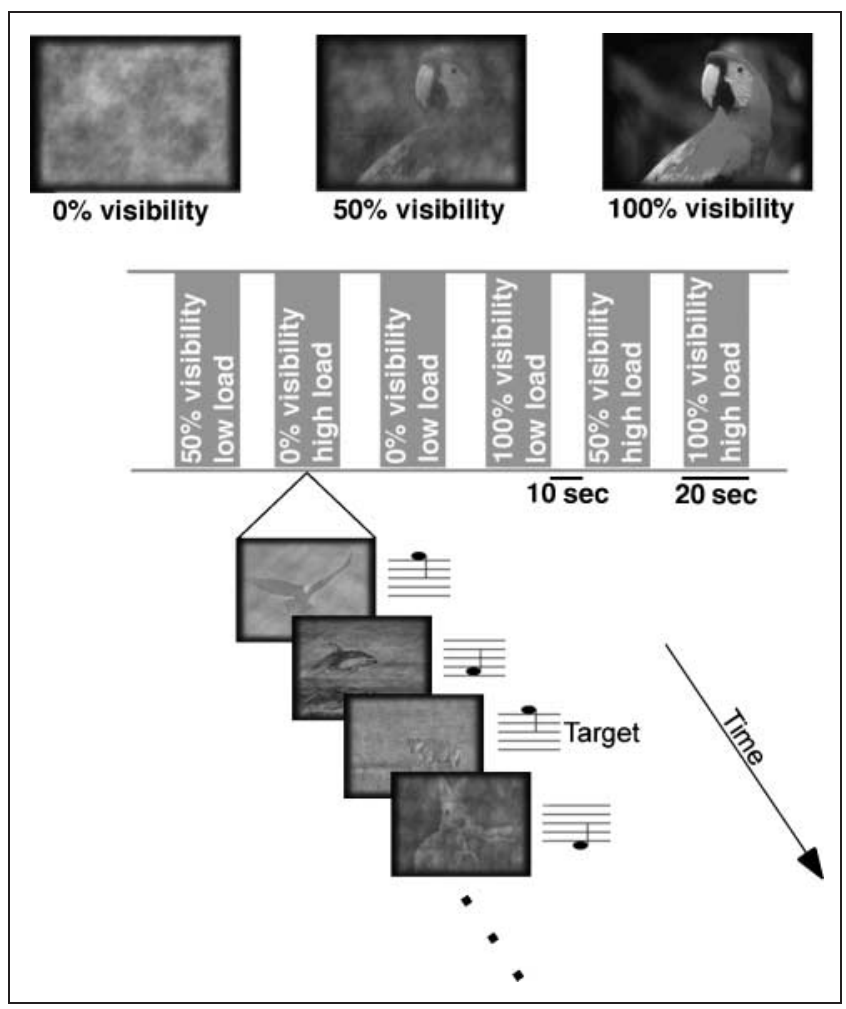

Figure 1. Experimental design. Participants performed an auditory matching task. Volunteers had to indicate the repetition of an identical tone by a button press. Object visibility (0\%, 50\%, and 100\%) and WM load (low and high) were combined to yield six conditions, which were repeated six times. 
The task was presented using a block design. Each block lasted $20 \mathrm{sec}$ and consisted of the presentation of 10 trials with consistent load and object visibility levels followed by a rest period in which a cue was presented for $10 \mathrm{sec}$, indicating which of the two tasks (1-back or 2-back WM) to perform next. In both tasks, participants heard a sequence of 10 tones presented for $1500 \mathrm{msec}$ each with an interstimulus interval (ISI) of $500 \mathrm{msec}$ (ISIs were jittered by $200 \mathrm{msec}$ in the behavioral/EOG experiment). Concurrently with the tones, task-irrelevant images were presented. Participants were instructed to hold fixation at a centrally presented cross and were instructed that the images were completely task-irrelevant and should be ignored. Although the images changed with each presented tone, the object visibility level was kept constant throughout each 10-trial block.

Participants practiced the task (for the fMRI experiment in the scanner, in order to adjust to the scanner noise) prior to the actual experiment until a satisfactory level of performance was attained.

Each of the two experimental sessions lasted 9 min and was followed by a short break. Reaction times (RTs) and error rates were recorded for all tasks.

Five pseudorandom orders of the conditions were created in which each condition was presented three times within a session. Images were pseudorandomized within the condition they were presented in to prevent repetition of images within and between consecutive blocks.

Tones were pseudorandomized so that every block contained 10 tones with three targets, one of which always occurred in last or second-to-last position of the 10-tone sequence in order to assure that the participants' attention remained on the task throughout each block.

\section{Recognition Task}

A recognition task was used to assess stimulus processing under the different load-dependent processing conditions.

Once the two sessions were completed, participants were asked to view all 60 of the presented images which had now been randomly intermixed with 60 new images; they were asked to indicate by button press for each image whether or not they had seen it previously. Each image was presented at 100\% object visibility for $2500 \mathrm{msec}$ with an ISI of $1000 \mathrm{msec}$. Data from one participant in the fMRI experiment were missing, as the responses were not collected due to hardware problems.

\section{Stimuli}

Visual stimuli consisted of 120 neutral images $(640 \times$ 480 pixels; 24-bit color depth) of natural scenes containing plants and animals taken from the International Affective Picture System (Lang, Öhman, \& Vaitl, 1988) and downloaded free of charge from the Internet (some sample visual stimuli can be seen in Figure 1). A random selection of 60 images was presented during the experiment and mixed with the remaining 60 images for the recognition task. Visibility of object information of the images was manipulated according to a method described by Rainer, Augath, Trinath, and Logothetis (2001). In order to embed the images smoothly into the background, and thus, reduce transient neural activity evoked by the outer edges of the images, the edges of the stimuli were smoothed with a 28-mm FWHM isotropic kernel. At the center of each image, a fixation cross was superimposed.

Auditory stimuli consisted of sine waves of various amplitudes $(200 \mathrm{~Hz}, 400 \mathrm{~Hz}, 600 \mathrm{~Hz}, 800 \mathrm{~Hz}$, and $1000 \mathrm{~Hz}$ both in the fMRI and behavioral control experiment), which were matched for length (1500 msec) and loudness (www. goldwave.com/). The amplitude steps were pretested and optimized in difficulty for the fMRI and EOG settings.

All stimuli were presented, responses were collected, and synchronization with the MR scanner was attained with the Presentation software (www.neuro-bs.com) running on a PC.

\section{Data Acquisition}

BOLD fMRI images were acquired with a 3-T Siemens Trio MR scanner with a gradient-echo EPI T2*-sensitive sequence. A whole-head acquisition was run with 42 slices of $2 \mathrm{~mm}$ thickness and $1 \mathrm{~mm}$ gap with an in-plane resolution of $3 \times 3 \mathrm{~mm}$, a TR of $2.45 \mathrm{sec}$, a TE of $25 \mathrm{msec}$, a flip angle of $80^{\circ}$, a field of view of $192 \times 192 \mathrm{~mm}$, and a $64 \times$ 64 matrix. A total of 228 images per run were acquired.

Visual stimuli were projected onto a screen at the back of the scanner bore and viewed via a mirror system. Auditory stimuli were delivered via headphones and responses given via a custom-made response box, both MR-compatible.

In the behavioral/EOG experiment, visual stimuli were viewed on a 15 -inch LCD monitor, at $1 \mathrm{~m}$ distance from the participants, and auditory stimuli were delivered via loud speakers.

Vertical and horizontal EOGs were recorded by electrodes, whose resistance was kept below $10 \mathrm{k} \Omega$. EOGs were amplified in the range from $0.03 \mathrm{~Hz}(=5 \mathrm{sec}$ time constant) to $120 \mathrm{~Hz}$ and A/D converted at a $500 \mathrm{~Hz}$ sampling rate.

\section{Behavioral Data Analysis}

Two-way (Load $\times$ Object Visibility) repeated measures ANOVAs were conducted on RTs, error, and recognition rates.

\section{fMRI Data Processing and Analysis}

SPM2 (www.fil.ion.ucl.ac.uk/spm) was used for image processing and statistical analysis. All volumes were realigned to that first acquired, spatially normalized to the standard EPI template (Evans et al., 1993), and smoothed with a 12-mm FWHM isotropic Gaussian kernel. 
At the single-subject level, each of the six conditions (1-back WM-0\% object visibility to 2-back WM-100\% object visibility) was statistically modeled as a box-car function, which was convolved with a hemodynamic response function. Using a general linear model (Friston et al., 1995), regression coefficients were obtained for each regressor (condition).

A second-level group analysis (paired-sample $t$ tests within the general linear modal) with intersubject variability as random effect was carried out, applying a nonsphericity correction. The analyses were strongly hypothesis-driven, thus small-volume corrections were applied (spheres with $10 \mathrm{~mm}$ radius) centered on peak coordinates of a similar previous study (MNI-left LOC: $-45-66$-9; right LOC: $42-66$-12; Glascher et al., 2007).

\section{EOG Data Processing and Analysis}

Vertical and horizontal EOGs were recorded referenced to the nose (Brain Vision System) and re-referenced on-line against each other. Data segments from $100 \mathrm{msec}$ prior to 900 msec poststimulus onset were created and checked for eye movement and blink artifacts (semiautomatic), zero-lines, out-of-range values, slow drifts, and fast amplitude shifts $>100 \mu \mathrm{V}$ within $2 \mathrm{msec}$ (all automatic). Breach of any of these criteria led to the rejection of the respective segment. All channels were filtered off-line at $35 \mathrm{~Hz}$. The mean activity of unsigned values from both EOG channels was calculated for the whole segment for each of the six conditions and entered into a two-way repeated measures ANOVA (Load $\times$ Object Visibility).

\section{RESULTS}

\section{Behavioral: fMRI Study}

All behavioral results are reported in Table 1 and illustrated in Figure 2. Both the error rates and RTs reflect only the load manipulation but not the different levels of object visibility. Thus, in both cases, the main effect of load was statistically highly significant, whereas the main effect of object visibility and the interaction of the two factors remained nonsignificant. The results for the recognition task were different, with both main effects and the interaction being highly significant. Following our predictions, the recognition rates reflected the impaired processing during encoding of the visual stimuli. Recognition increased with increasing object visibility and was impaired under high, compared to low, load, especially so the higher the object visibility was.

\section{Functional Magnetic Resonance Imaging}

Effects of Auditory WM Load on Processing in the LOC

As anticipated, the fMRI random effects (RFX) group data show a main effect of object visibility, increasing object visibility leading to increased BOLD signal intensity in the LOC ROI (see "fMRI Data and Analysis" for the choice of the ROI) [left (MNI -36-69-9): $t(55)=9.76, p_{\mathrm{FWE}}<$ .001 ; right (MNI $\left.45-57-15): t(55)=8.38, p_{\mathrm{FWE}}<.001\right]$ and a main effect of WM load, high WM load resulting in decreased fMRI signal in the LOC ROIs [left (MNI -42 $-75-6): t(55)=3.63, p_{\mathrm{FWE}}=.01 ;$ right $(\mathrm{MNI} 45-57$ $\left.-15): t(55)=3.24, p_{\mathrm{FWE}}=.03\right]$. An interaction of the WM load and the object visibility level of the task-irrelevant images was observed in the left LOC. The increase in BOLD signal across object visibility levels interacted with the WM load, resulting in a phasic modulation, that is, the BOLD signal increase with object visibility was less strong under concomitant high WM load than it was under low WM load [left $(\mathrm{MNI}-48-660): t(55)=3.06, p_{\mathrm{FWE}}=.04$; Figure 3). Further exploratory analysis of the whole brain at $p_{\text {FDR }}<.05$ revealed no further activated clusters for this interaction.

\section{Effects of Auditory WM Load on Processing in Other Visual Cortices}

In order to be able to compare the results for the LOC with the activation in other visual cortices, we ran further analyses on all areas active in the main effect of Object Visibility. The interaction WM Load $\times$ Object Visibility did not reach significance elsewhere in visual cortices, but the main effect of WM Load did reach statistical significance in several visual areas, which are listed in Table 2.

\section{Effects of Auditory WM Load Not Scaled by Object Visibility in Visual Cortices}

In addition to visual areas in which the WM load effect was scaled by the object visibility, some areas were found to be unaffected by the object visibility. These areas are listed in Table 3.

\section{Behavioral: Control Experiment with EOG Recordings}

Because eye movements could not be recorded in the fMRI scanner, the possibility had to be considered that the stronger BOLD activation observed for the higher object visibility levels was, at least in part, related to increased eye movements with increasing background object visibility. Because receptive fields in the LOC are relatively large (Larsson \& Heeger, 2006), modulation of activity in this area is unlikely to be explained solely by eye movements. Nevertheless, we repeated the experiment outside the fMRI scanner with eye-movement monitoring and otherwise identical stimuli (auditory stimuli were optimized for use outside the MR-scanner; see section "Stimuli"). Table 1 and Figure 2 show that all behavioral results broadly conformed to those of the fMRI study. In addition, vertical and horizontal EOGs were recorded and we were able to confirm that none of the conditions differed from each other (Table 1 and Figure 2). 
Table 1. Behavioral Results for the fMRI and Control Experiments

\begin{tabular}{|c|c|c|c|c|}
\hline & \multicolumn{2}{|c|}{$f M R I$} & \multicolumn{2}{|c|}{ Control } \\
\hline & $F(d f)$ & $p$ & $F(d f)$ & $p$ \\
\hline \multicolumn{5}{|l|}{ Errors } \\
\hline Load & $66.52(1,11)$ & $<.001$ & $25.12(1,11)$ & $<.001$ \\
\hline Visibility & $0.25(2,22)$ & .781 & $0.55(2,22)$ & .586 \\
\hline Load $\times$ Visibility & $0.40(2,22)$ & .673 & $0.21(2,22)$ & .810 \\
\hline \multicolumn{5}{|l|}{$R T s$} \\
\hline Load & $40.00(1,11)$ & $<.001$ & $32.93(1,11)$ & $<.001$ \\
\hline Visibility & $0.08(2,22)$ & .920 & $6.59(2,22)$ & .006 \\
\hline Load $\times$ Visibility & $0.87(2,22)$ & .434 & $2.40(2,22)$ & .114 \\
\hline \multicolumn{5}{|l|}{ Recognition } \\
\hline Load & $9.57(1,10)$ & .011 & $10.71(1,11)$ & .007 \\
\hline Visibility & $73.36(2,20)$ & $<.001$ & $51.29(2,22)$ & $<.001$ \\
\hline Load $\times$ Visibility & $12.17(2,20)$ & $<.001$ & $5.83(2,22)$ & .009 \\
\hline \multicolumn{5}{|l|}{$V E O G$} \\
\hline Load & & & $1.11(1,11)$ & .315 \\
\hline Visibility & & & $1.87(2,22)$ & .178 \\
\hline Load $\times$ Visibility & & & $0.13(2,22)$ & .576 \\
\hline \multicolumn{5}{|l|}{$H E O G$} \\
\hline Load & & & $0.02(1,11)$ & .892 \\
\hline Visibility & & & $0.86(2,22)$ & .436 \\
\hline Load $\times$ Visibility & & & $1.27(2,22)$ & .300 \\
\hline
\end{tabular}

Two-way repeated measures ANOVA $p, F$, and $d f$ values for the fMRI and behavioral control experiments. All effects found in the fMRI experiment were closely replicated in the control study.

Therefore, it is unlikely that the observed increase in BOLD activation in the LOC with increasing object visibility was caused merely by differences in eye-movement patterns between the different levels of object visibility.

\section{DISCUSSION}

In this experiment, the interference effect of a relevant WM task on the processing of task-irrelevant stimuli was examined. The possibility needed to be accounted for that if both the relevant and the irrelevant stimuli were presented to the same modality, these would already compete for resources at a perceptual level, thus leading to perceptual, rather than WM load, interference. For this reason, we closely replicated a previous study (Rose et al., 2005), but presented our task-relevant and task-irrelevant stimuli to different modalities. WM load induced interference was anticipated to follow a pattern contrary to that posited by load theory (Lavie et al., 2004) and in line with previous findings (Bingel et al., 2007; Glascher et al., 2007; Rose et al., 2005) and a more generalized view of load effects. Both our behavioral and our fMRI results are in close correspondence with the results of the previous study (Rose et al., 2005), and so we can conclude that, in both cases, the WM, rather than perceptual aspects of the relevant task, interfered with the processing of task-irrelevant stimuli in the LOC.

The fMRI results confirm that the manipulation of object visibility was successful in the LOC, as activity in this region increased monotonically with increasing object visibility. In addition, the manipulation of the relevant auditory task load was successful and followed our prediction: Processing of task-irrelevant stimuli in the LOC was reduced under high, compared to low, concomitant WM load. WM load and object visibility interacted in a task-variant manner in the left LOC, revealing a steeper 


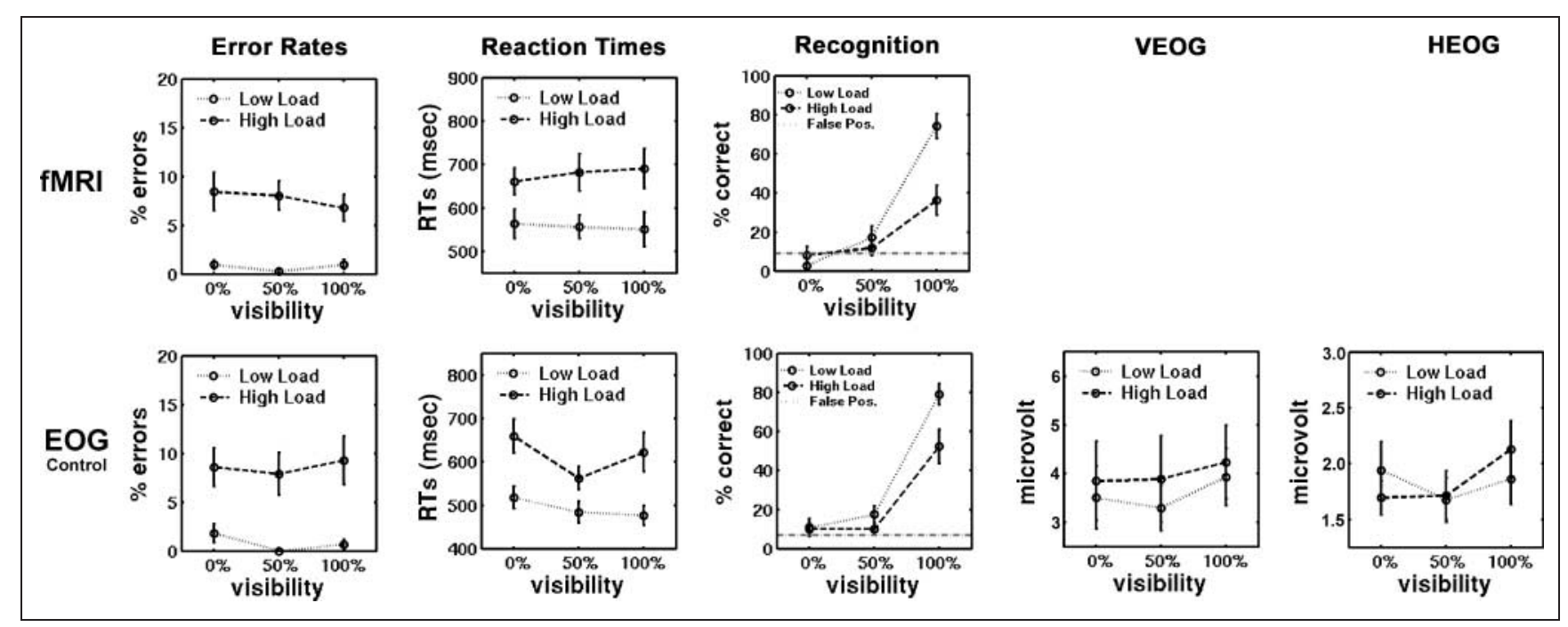

Figure 2. Behavioral results of the fMRI and behavioral control study. Percent error rates, RTs, and recognition rates for each condition in the fMRI and behavioral control experiment as well as vertical and horizontal EOGs for each condition in the behavioral control experiment. Error bars show standard errors of the mean. The error rates, RTs, and recognition rates visibly confirm that the task manipulation of WM load was successful in both experiments. The recognition rates of both studies further confirm that the manipulation of increasing object visibility was successful. The EOGs illustrate that the different conditions did not result in eye-movement differences between the conditions, and thus could not account for the behavioral or fMRI results.

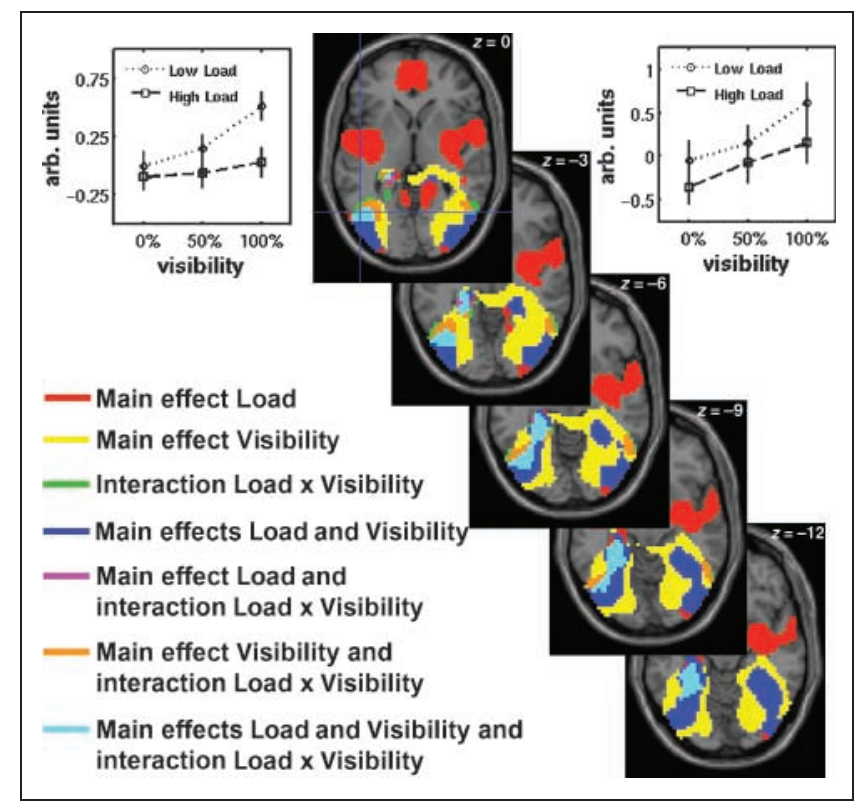

Figure 3. BOLD effects in lateral occipital cortex. Activation clusters for the main effects of load and object visibility, the interaction of load and object visibility, and all combinations of these effects are shown on five axial slices of a single-subject template brain. For illustrative purposes, only the main effects of load and object visibility were thresholded at $p_{\mathrm{FDR}} \leq .05$, the interaction of load and object visibility was inclusively masked by the main effect of object visibility ( $p \leq .05$ uncorrected) and is shown at $p \leq .05$, uncorrected. A cluster threshold of 100 voxels was applied to all contrasts and all images were binarized. Mean beta weights and standard errors of the mean for all conditions are plotted at the top of the figure. On the left side, the interaction effect found in the left hemisphere is plotted; on the right side, the plot displays the main effects of load and object visibility found in the right hemisphere. slope for object visibility under low than high WM load. This influence is also seen in the recognition rates of the surprise recognition task at the end of the experiment. The high auditory WM load resulted in a suppressed recognition of the irrelevant images, dependent on the object visibility. This is in accord with the fMRI results that indicated reduced processing of the images in the LOC under high WM load.

In addition to the interaction effect observed in the LOC, a widespread general suppression of activation that did not interact with object visibility was observed in visual cortices under high compared to low WM load. Although we did not make any explicit predictions about activations in other visual areas, this result is unsurprising. Our stimuli were specifically chosen to elicit an optimal response from the LOC, hence, an interaction of WM with processing in

Table 2. Visual Cortical Areas in Which a Main Effect of Load and a Main Effect of Object Visibility Were Found

\begin{tabular}{lcrc}
\hline Area & $x y z(M N I)$ & $p_{F D R}$ & $t(55)$ \\
\hline Lateral occipital cortex (L) & $-48-816$ & $<.001$ & 5.46 \\
Lateral occipital cortex (L) & $-42-909$ & .001 & 5.20 \\
Parahippocampal gyrus (L) & $-27-36-9$ & .001 & 4.74 \\
Temporal occipital fusiform & $36-45-15$ & .001 & 4.83 \\
$\quad$ cortex (R) & $15-9039$ & .002 & 4.26 \\
Occipital pole (R) & &
\end{tabular}

The main effect of load was inclusively masked by the main effect of object visibility ( $p \leq .001$, uncorrected) and effects are reported at $p_{\mathrm{FDR}} \leq .05$. 
Table 3. Visual Cortical Areas in Which a Main Effect of Load, but No Main Effect of Object Visibility, Was Found

\begin{tabular}{lccc}
\hline Area & $x y z(M N I)$ & $p_{F D R}$ & $t(55)$ \\
\hline Occipital pole (L) & $-12-9039$ & .002 & 4.11 \\
Occipital pole (R) & $36-960$ & .005 & 3.63 \\
Lateral occipital cortex (R) & $42-87-15$ & .040 & 2.45 \\
Lateral occipital cortex (L) & $-54-726$ & .006 & 3.49 \\
Lateral occipital cortex (L) & $-48-666$ & .014 & 3.03 \\
Occipital pole (L) & $-33-990$ & .018 & 2.89 \\
Middle temporal gyrus (R) & $66-549$ & .023 & 2.76 \\
Lateral occipital cortex (R) & $57-696$ & .030 & 2.60 \\
\hline
\end{tabular}

The main effect of load was exclusively masked by the main effect of object visibility ( $p \leq .001$, uncorrected) and effects are reported at $p_{\mathrm{FDR}} \leq .05$.

this area was predicted. For all other visual areas, no more than a general WM load effect should be anticipated.

In the right LOC, a similar general suppression of activation under high WM was observed, but no interaction with object visibility, as observed in the left LOC. Although it is theoretically possible that the left and right LOC are modulated by different mechanisms, to our knowledge, no evidence exists to support such a functional dichotomy. More likely, as a consequence of a relatively modest sample size, statistical power was lacking, and thus, only a generalized suppression effect, but no interaction with visibility, was observed in the right LOC.

\section{Effects of Different Types of Load}

In addition to the finding that a relevant WM task of high load attenuates the processing of irrelevant visual stimuli in the LOC (Rose et al., 2005), further studies have (1) modulated concurrent experimentally induced pain and found this to have a similar interfering effect on LOC processing as WM load does (pain reduces processing of task-irrelevant stimuli; Bingel et al., 2007); or (2) modulated concurrent emotional valence of the images and have found this to have a modulating effect on LOC processing, which is in line with perceptual load theory (negative valence increases processing; Glascher et al., 2007). All of the above-named results, as well as those reported in this article, follow the pattern of load interference described for perceptual rather than cognitive load (Lavie et al., 2004; Lavie, 1995). Thus, as outlined above, the possibility may be considered that the interference effects caused by perceptual and WM load are more similar than suggested by Lavie et al. (2004) and Lavie (1995) and that, in addition, other types of load, for instance, pain and emotional valence, may also cause similar interference effects. This possibility would posit that all types of high load of a relevant task engage large proportions of limited attentional resources, and thus, render them unavailable for the processing of irrelevant stimuli; the processing of irrelevant stimuli consequently suffers from this dearth of resources.

A related theory of attentional interference is multiple resource theory, according to which different tasks draw on different resources (e.g., verbal vs. spatial WM), and thus, only tasks that require the same resources should interfere with each other's processing (Navon, Gopher, Chillag, \& Spitz, 1984; Gopher, Brickner, \& Navon, 1982; Isreal, Chesney, Wickens, \& Donchin, 1980; Wickens \& Kessel, 1980). In a series of dual-task experiments, Kim, Kim, and Chun (2005)) have, for example, shown that limited-capacity resources can be tied up in a WM task and are subsequently unavailable to the processing of either targets or distractors in a Stroop task (Stroop, 1935). Critically, only WM tasks that draw on the same processing resources as the targets or distractors of the Stroop task have an effect on the performance on the Stroop task. In the current experiment, we did not compare different resources, so possibly another task would have interfered with irrelevant stimulus processing yet more strongly than our WM task. However, interference was observed and cannot be explained adequately either by load theory or by multiple resource theory. Notably, however, multiple resource theory postulates only one type of interference for all types of resources: A relevant task engages resources, which are consequently unavailable to a secondary task, whose processing suffers from the lack of resources. In this respect, we concur with multiple resource theory.

In line with our findings, other groups have also reported reduced distractor processing under high compared to low WM load. In SanMiguel, Corral, and Escera's (2008) experiment, participants performed either a 0-back or a 1-back visual WM task while concurrently being presented with task-irrelevant repetitive standard tones and occasional novel environmental sounds. In a similar study, participants indicated whether repetitive standard tones and occasional novel sounds were of a long or short duration (Berti \& Schroger, 2003). The difficulty of the task was varied by performing the duration judgment on the current (0-back WM) or the previously heard (1-back WM) sound. In both studies, distraction by the sounds was reduced as indexed by higher hit rates, faster RTs, and attenuation of the novelty-P3 and the reorienting negativity ERP components in response to novel sounds. The early ERP mismatch negativity was unaffected by WM load. The authors interpreted their findings in light of a recent suggestion that the WM system protects current task-related processing from potentially interfering information by suppressing the processing of such input. The preattentive change detection system, however, is unaffected by WM, allowing full preattentive scanning of the environment (Postle, 2006). In line with such a suggestion, Spinks, Zhang, Fox, Gao, and Hai Tan (2004) used a low or high demand subtraction task paired with irrelevant words that were presented in one of three languages in which participants had a high, intermediate, or no level of proficiency. When the central executive was strongly engaged by the 
high load condition, it was less prone to attentional capture and thus interference from the task-irrelevant stimuli was low. However, the more novel the irrelevant words were, the more they managed to capture attention even under high load.

Note that we do not mean to suggest that one single mechanism or cortical area mediates the effects of all different types of load or that the strength of the effects of different types of load should be the same. Rather, we propose that the general pattern of effects should be the same, regardless of the types of load that caused them: High load of a relevant task reduces the resources available for processing stimuli of an irrelevant task.

\section{A Single Load Mechanism}

Although the above named studies appear to paint a coherent picture of the effects of various types of load, our results stand in contrast to previous studies, which show an effect of WM load contrary to ours (Lavie et al., 2004; de Fockert et al., 2001). A resolution to this apparent contradiction in results, however, can be given by positing a single mechanism, which can account for the differences in results. In the studies of Lavie et al. (2004) and de Fockert et al. (2001), participants held numbers in WM while performing a selective attention task. High WM load was found to impair performance on the selective attention task, leading to the conclusion that distractors had been processed more under high than low WM load. That is, WM load impaired attentional selection, and thus, the suppression of irrelevant stimuli was less effective. In our experiment, on the contrary, irrelevant stimulus processing was "directly" suppressed by WM load, as opposed to WM load affecting selective attention and selective attention, in turn, affecting the processing of the irrelevant stimuli. Hence, all cited studies show that WM load reduces resources available for another task: attentional selection in Lavie et al.'s and de Fockert et al.'s and ventral visual processing in our study.

\section{Conclusions}

In this experiment, we studied the interference effect of WM load on the processing of task-irrelevant stimuli and found this interference to be similar to that of perceptual load. We suggest that in previous studies similar results were obtained but were interpreted differently due to the use of a dual-task paradigm in which selective attention, rather than the actual processing of task-irrelevant stimuli, was affected. It is therefore proposed that the same or a similarly functioning mechanism governs the effects of both perceptual and WM load: High load of a relevant task engages limited attentional resources, which are consequently unavailable for the processing of taskirrelevant stimuli. By comparing our current results with previously published findings, we furthermore deduce that other types of load, such as pain and emotional va- lence, follow a similar pattern of interference, and thus, an inclusion of these in a theoretical framework may be found to be useful.

\section{Acknowledgments}

This research was supported by grants from the Volkswagenstiftung (C. B.), BMBF (C. B., J. K., M. R., M. M.), and the DFG (C. B., M. R.); additionally, the preparation was supported by a grant from the BBSRC (J. K.). We thank the Physics and Methods group at NeuroImage Nord, Hamburg for help with MR scanning, Penelope Klemen for language editing, and Chris Chambers for helpful comments on an earlier draft of this manuscript.

Reprint requests should be sent to Dr. Jane Klemen, CUBRIC, School of Psychology, Tower Building, Park Place, Cardiff CF10 3AT, UK, or via e-mail: klemenj@cardiff.ac.uk.

\section{REFERENCES}

Berti, S., \& Schroger, E. (2003). Working memory controls involuntary attention switching: Evidence from an auditory distraction paradigm. European Journal of Neuroscience, 17, 1119-1122.

Bingel, U., Rose, M., Glascher, J., \& Buchel, C. (2007). fMRI reveals how pain modulates visual object processing in the ventral visual stream. Neuron, 55, 157-167.

Broadbent, D. E. (1958). Perception and communication. London: Pergamon Press.

de Fockert, J. W., Rees, G., Frith, C. D., \& Lavie, N. (2001). The role of working memory in visual selective attention. Science, 291, 1803-1806.

Deutsch, J. A., \& Deutsch, D. (1963). Attention: Some theoretical considerations. Psychological Review, 70, 51-61.

Duncan, J. (1980). The locus of interference in the perception of simultaneous stimuli. Psychological Review, 87, 272-300.

Evans, A. C., Collins, D. L., Mills, D. R., Brown, E. D., Kelly, R. L., \& Peters, T. M. (1993). 3D statistical neuroanatomical models from 305 MRI volumes. Proceedings of the IEEE Nuclear Science Symposium and Medical Imaging, 1-3, 1813-1817.

Friston, K. J., Holmes, A. P., Poline, J. B., Grasby, P. J., Williams, S. C., Frackowiak, R. S., et al. (1995). Analysis of fMRI time-series revisited. Neuroimage, 2, 45-53.

Glascher, J., Rose, M., \& Buchel, C. (2007). Independent effects of emotion and working memory load on visual activation in the lateral occipital complex. Journal of Neuroscience, 27, 4366-4373.

Gopher, D., Brickner, M., \& Navon, D. (1982). Different difficulty manipulations interact differently with task emphasis: Evidence for multiple resources. Journal of Experimental Psychology: Human Perception and Performance, 8, 146-157.

Grill-Spector, K., Kushnir, T., Hendler, T., Edelman, S., Itzchak, Y., \& Malach, R. (1998). A sequence of object-processing stages revealed by fMRI in the human occipital lobe. Human Brain Mapping, 6, 316-328.

Grill-Spector, K., Kushnir, T., Hendler, T., \& Malach, R. (2000). The dynamics of object-selective activation correlate with recognition performance in humans. Nature Neuroscience, 3, 837-843.

Isreal, J. B., Chesney, G. L., Wickens, C. D., \& Donchin, E. (1980). P300 and tracking difficulty: Evidence for multiple resources in dual-task performance. Psychophysiology, 17, 259-273. 
Kim, S. Y., Kim, M. S., \& Chun, M. M. (2005). Concurrent working memory load can reduce distraction. Proceedings of the National Academy of Sciences, U.S.A., 102, 16524-16529.

Lang, P. J., Öhman, A., \& Vaitl, D. (1988). The International Affective Picture System (Slides). Gainsville, FL: Center for Research in Psychophysiology, University of Florida.

Larsson, J., \& Heeger, D. J. (2006). Two retinotopic visual areas in human lateral occipital cortex. Journal of Neuroscience, 26, 13128-13142.

Lavie, N. (1995). Perceptual load as a necessary condition for selective attention. Journal of Experimental Psychology: Human Perception and Performance, 21, 451-468.

Lavie, N., Hirst, A., de Fockert, J. W., \& Viding, E. (2004). Load theory of selective attention and cognitive control. Journal of Experimental Psychology: General, 133, 339-354.

Lavie, N., \& Tsal, Y. (1994). Perceptual load as a major determinant of the locus of selection in visual attention. Perception \& Psychophysics, 56, 183-197.

Malach, R., Reppas, J. B., Benson, R. R., Kwong, K. K., Jiang, H., Kennedy, W. A., et al. (1995). Object-related activity revealed by functional magnetic resonance imaging in human occipital cortex. Proceedings of the National Academy of Sciences, U.S.A., 92, 8135-8139.

Navon, D., Gopher, D., Chillag, N., \& Spitz, G. (1984). On separability of and interference between tracking dimensions in dual-axis tracking. Journal of Motor Behaviour, 16, 364-391.

Norman, D. A. (1968). Towards a theory of memory and attention. Psychological Review, 75, 522-536.

Postle, B. R. (2006). Working memory as an emergent property of the mind and brain. Neuroscience, 139, 23-38.

Rainer, G., Augath, M., Trinath, T., \& Logothetis, N. K. (2001). Nonmonotonic noise tuning of BOLD fMRI signal to natural images in the visual cortex of the anesthetized monkey. Current Biology, 11, 846-854.

Rees, G., Frackowiak, R., \& Frith, C. (1997). Two modulatory effects of attention that mediate object categorization in human cortex. Science, 275, 835-838.

Rees, G., Frith, C. D., \& Lavie, N. (1997). Modulating irrelevant motion perception by varying attentional load in an unrelated task. Science, 278, 1616-1619.

Reinholz, J., \& Pollmann, S. (2005). Differential activation of object-selective visual areas by passive viewing of pictures and words. Brain Research, Cognitive Brain Research, 24, 702-714.

Rose, M., Schmid, C., Winzen, A., Sommer, T., \& Buchel, C. (2005). The functional and temporal characteristics of top-down modulation in visual selection. Cerebral Cortex, 15, 1290-1298.

SanMiguel, I., Corral, M. J., \& Escera, C. (2008). When loading working memory reduces distraction: Behavioral and electrophysiological evidence from an auditory-visual distraction paradigm. Journal of Cognitive Neuroscience, 20, 1131-1145.

Schwartz, S., Vuilleumier, P., Hutton, C., Maravita, A., Dolan, R. J., \& Driver, J. (2005). Attentional load and sensory competition in human vision: Modulation of fMRI responses by load at fixation during task-irrelevant stimulation in the peripheral visual field. Cerebral Cortex, 15, 770-786.

Spinks, J. A., Zhang, J. X., Fox, P. T., Gao, J. H., \& Hai Tan, L. (2004). More workload on the central executive of working memory, less attention capture by novel visual distractors: Evidence from an fMRI study. Neuroimage, 23, 517-524.

Stroop, R. J. (1935). Studies of interference in serial verbal reactions. Journal of Experimental Psychology, 18, 643-662.

Treisman, A. M., \& Geffen, G. (1967). Selective attention: Perception or response? Quarterly Journal of Experimental Psychology, 19, 1-17.

Wickens, C. D., \& Kessel, C. (1980). Processing resource demands of failure detection in dynamic systems. Journal of Experimental Psychology: Human Perception and Performance, 6, 564-577. 